\title{
Electronic Medical Records: Friends or Foes?
}

\author{
Michael D. Ries MD
}

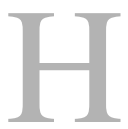
istorically, hospitals maintained medical records with paper charts and printed radiographs, which were then stored and maintained at outpatient practices

Note from the Editor-in-Chief: We are pleased to present to readers of Clinical Orthopaedics and Related Research ${ }^{\mathbb{B}}$ another installment of Clinical Faceoff, a regular feature. This section is a point-counterpoint discussion between recognized experts in their fields on a controversial clinical or nonclinical issue. We welcome reader feedback on all of our columns and articles; please send your comments to eic@clinOrthop.org.

The author certifies that he, or a member of his immediate family, has no funding or commercial associations (eg, consultancies, stock ownership, equity interest, patent/ licensing arrangements, etc) that might pose a conflict of interest in connection with the submitted article.

All ICMJE Conflict of Interest Forms for authors and Clinical Orthopaedics and Related Research editors and board members are on file with the publication and can be viewed on request.

The opinions expressed are those of the writers and do not reflect the opinion or policy of $\operatorname{CORR}^{\mathbb{R}}$ or the Association of Bone and Joint Surgeons ${ }^{\circledR}$.

M. D. Ries MD ( $\square)$

Tahoe Fracture and Orthopaedic Clinic, 973 Mica Dr., Carson City, NV 89705, USA

e-mail: riesm@orthosurg.ucsf.edu and hospitals. Current technology permits conversion of these records to electronic media, and increasingly, physicians are documenting their clinical activities directly into an electronic medical record (EMR). However, many aspects of implementation and use of EMRs are controversial. For this Clinical Faceoff, I have invited two colleagues with expertise and experience in the implementation of an EMR in clinical practice. Both have strong academic records, but clearly hold some divergent points of view with respect to the use of EMRs in clinical practice.

Thomas C. Barber MD is an Associate Physician-in-Chief at the Oakland Medical Center, and has served as Chair of the Orthopaedic Chiefs for the Kaiser Permanente Medical Group. He was also Chair of the CyberKaiser Development team, and has been active in the American Academy of Orthopaedic Surgeons (AAOS) initiatives in this area. He also has served as Chair of the AAOS Board of Councilors and a member of AAOS committee for a National Total Joint Registry, Committee on Health Care Delivery, Committee on Prevention of Medical Errors, and AAOS Representative for the Practicing Physician Advisory Council to the National Committee on Quality Assurance.
Sigurd Berven MD is a professor of Spine Surgery at the University of California, San Francisco. He has published more than 120 peer-reviewed journal articles with a focus on outcomes research and evidence based medicine in spine surgery. He served as the American Orthopaedic Association symposium director for Value in Healthcare, and on the North American Spine Committee for Value in healthcare.

Michael D. Ries MD: Who benefits the most from the cost savings produced by an EMR (hospitals, insurance companies, physicians, or patients)? Why do you think so?

Sigurd Berven MD: Evidence that an EMR will produce measurable cost savings remains limited. The USD 27 billion investment in health information technology made by our federal government through the American Recovery and Reinvestment Act of 2009 is intended to reduce healthcare cost through reduction of overutilization of services and adverse events [5]. However, a report by the Congressional Budget Office [8] raised significant concerns about EMRs leading to an increase in healthcare spending through reducing the efficiency of care, promoting overutilization of unnecessary services, and reducing the ability of the 
clinical care pathways to respond to patient and provider preferences. In a systematic review of health information technologies, cost-effectiveness modeling has demonstrated that the EMR may require more than 10 years before cost savings exceed investment costs [16]. In the absence of significant improvement in evidence-based approach to care, clinical care pathways based upon patient-centered outcomes, and a value-based approach to clinical care, implementation of an EMR alone is unlikely to produce cost savings or enhance value [17]. Insurance companies, the Centers for Medicare \& Medicaid Services (CMS), and our healthcare budget will realize the most direct benefits of cost saving through reducing overutilization. Federal incentives for EMR utilization and meaningful use may benefit hospitals and physicians who are early and effective adopters of the EMR, but this benefit is not a direct reflection of cost savings. The promise that implementation of an EMR will lead to measurable benefits reaching the most important and central stakeholder, the patient, is remote. Benefits will only occur if cost saving is sustained, which can only happen after hospitals and third-party payers benefit from the early stages of cost saving.

Thomas C. Barber MD: When an EMR is correctly implemented, cost savings will accrue to hospitals, physician practices, and insurers. The key point is that an EMR does not simply replace paper, it enhances the systems you have in place to manage patients. Cost savings accrue for physician practices if they abandon dictation in favor of "smart phrases" (typing shortcuts) in the EMR, and if they take the time to set up excellent templates for common procedures and consultations. A large survey found that $79 \%$ of practices perform more efficiently after implementation of an EMR [3]. Benefits to hospitals are estimated between USD 37 million and USD 59 Million in 5 years [3]. This significant savings derives from decreased medical records expense in storing and sorting the paper charts.

Dr. Ries: The EMR is intended to reduce paperwork, improve efficiency, and reduce healthcare costs. To what degree do you believe those potential benefits will be realized?

Dr. Barber: These benefits will be realized in time, and only to the extent that you can change your practice and the systems in your offices to meet the challenges of EMR implementation. Clearly paperwork is reduced, but to get maximum reduction in paper you need the ability to share your EMR records with your hospital electronically, connect with labs and radiology providers to receive results electronically, and connect with insurers electronically for claims and adjudication of claims.
Efficiency is only improved if your practice changes in the EMR area.

The efficiencies result when you do things differently. They do not result just from implementing an EMR. The same is true about cost reduction. Recognize though, that cost reductions accrue to the entire healthcare system, not to your office. An example is drugdrug interaction screening. Your EMR can pick up incompatible drugs so that you do not write a prescription in error. That prevents long-term problems or inpatient care that might have resulted from a drug reaction. The care of the patient is better, and overall costs are reduced, but your office costs may be the same or even higher. We have to remember that better quality care results in lower cost and the EMR is an enabler of better quality care. Where good integration exists between different specialties, the hospital, and the pharmacy improved quality and reduced costs are readily realized.

Dr. Berven: Reducing paperwork, improving efficiency, and reducing costs are important goals of the EMR, and all clinicians share the hope that an EMR may lead to these endpoints. Unfortunately, experience and data both support the conclusion that these goals are unlikely to be reached with present EMR systems alone. The EMR clearly generates more paper use. The printing of redundant and irrelevant information is routine and embedded 


\section{Clinical Faceoff}

into the work flow of EMR systems. There is good evidence that paper volume and paperwork are both increased with present EMR systems [18]. The most important mechanism by which the EMR may improve a physician's efficiency is through the use of templates. Templates invite inaccurate data entry, documentation of testing, and procedures that may not be performed, and fraud. The potential efficiencies of a template-based EMR system may well be negated by significant negative effects of the EMR on medical record accuracy and on patient privacy [14]. The mechanism by which an EMR may contribute to reducing costs is in reducing duplication of tests, and improving adherence to evidence-based protocols for care. Unfortunately, there is significant evidence [8] that EMR is more likely to increase healthcare spending through reducing efficiency of care, promoting overutilization of unnecessary services, and forcing the clinical care provider to respond to pathways that are not patient-centered.

Dr. Ries: The EMR provides electronic access for a large number of healthcare providers, administrators, and information technology personnel to view confidential patient records. How might we mitigate the risks to patient confidentiality associated with the involvement of these parties?
Dr. Berven: The EMR facilitates access to healthcare information, and presents a significant risk of inappropriate and unintended access to private healthcare information. One of the major travesties of our healthcare system is the ability of insurers to deny coverage of medical conditions based upon preexisting status. Inappropriate and unauthorized access to sensitive medical information may be facilitated for employers, insurance companies, and others. Widespread access to sensitive medical information creates a significant risk to patient privacy and to the confidentiality of medical data. In a crosssectional population study, the majority of respondents expressed significant concern regarding privacy and security of healthcare information in an EMR [9]. Protection of privacy of patient information is an important priority for EMR systems. A systematic review of security of EMR identified significant variability and inadequacies of techniques for privacy protection [10]. Modalities to mitigate risks to patient confidentiality include training of healthcare providers and administrators, encryption of medical records, sophisticated password protection for access to information, and limited storage of data in single repositories. Protecting health information privacy remains a major challenge to the ability of the EMR to effectively and efficiently permit exchange of health information between providers, researchers, and facilities.

Dr. Barber: The fear of breach of confidentiality with electronic systems is fed by public concerns, and by the press. In many ways, protection of privacy is enhanced, since we can actually track access to medical records in a real time basis. There are many examples of hospital and health system employees being fired or charged due to inappropriate access to electronic medical records. The system logs provide all the evidence needed in these cases. Prior to EMR, someone would have to view you looking at the wrong chart in order to alert management of inappropriate access. Enforcement of the law and of professionalism standards is much easier with the new systems [2].

That being said, access to all aspects of the medical records makes more information available to many more people, so risks of privacy breaches has increased. New protections such as requiring encryption on laptops and USB drives of medical system employees can mitigate the risk. We have the technological tools to prevent access, but problems can occur when people do not implement those solutions.

Dr. Ries: Who is required to implement an EMR in their clinical practice, and what happens if a physician chooses not to use an EMR? 


\section{Clinical Faceoff}

Dr. Barber: Medicare is requiring all physicians caring for Medicare patients to not only install an EMR system, but also to use it in a meaningful way. This is what the meaningful use criteria are all about. Payments of up to USD 44,000 per doctor during a number of years are available to providers for meeting meaningful use. Starting in 2014, any physician not meeting meaningful use criteria will be subject to penalties. The penalty is $1 \%$ of Medicare payments in 2015 (based on 2014 data), $2 \%$ in 2016, and $3 \%$ thereafter [7].

Presently, 50\% of all Medicare physicians have submitted meaningful use attestations [19]. Approximately $20 \%$ of orthopedic surgeons have submitted for use, yet 55\% have EMRs, according to survey data from the American Academy of Orthopaedic Surgeons [1]. Anyone not meeting meaningful use for at least 3 months of 2014 will be subject to penalties in 2015. Do not wait. Qualify now.

Dr. Berven: The EMR mandate is implemented through a series of incentives and penalties. Since 2011, the American Recovery and Reinvestment Act provided incentive payments through Medicare for implementing EMR systems. In parallel, CMS implemented a series of progressive penalties that would ensure compliance of any provider who cares for Medicare or Medicaid patients. Specifically, in 2015,
Medicare reimbursements will be reduced by $1 \%$. The deduction rate increases in subsequent years by $2 \%$ in $2016,3 \%$ in $2017,4 \%$ in 2018 , and up to $95 \%$ depending on future adjustments [7]. A pathway for other thirdparty payers to incentivize and penalize the use of an EMR and clinical care pathways are in development, and clearly will have a major influence on the behavior of orthopaedic practices. The effectiveness of financial-based incentives on influencing physician behavior has been reported variably, and there are many examples of isolated financial incentives having a limited role in changing physician practices [13]. Clear and empirical evidence of effectiveness of the EMR in improving practice efficiency, healthcare cost, and quality of care will be far more compelling than financial incentives in influencing physician behavior. Punitive financial incentives in the absence of evidence to support the EMR may lead to significant limitations in access to care for our elderly and disabled patients. The most effective way to incentivize orthopaedic surgeons to adopt an EMR will be to develop a system that has compelling evidence that the EMR will improve quality of care. The evidence to that widespread implementation of an EMR will improve efficiency and quality of care remains elusive.
Dr. Ries: What pitfalls might exist with the wider use of outpatient, inpatient, and operative note templates? To what degree should we be concerned about the potential for billing fraud, medical error (from careless use of templates), or other issues related to broader implementation of templates?

Dr. Berven: Templates and prepopulated datasets embedded into the EMR dissociate the recording of information from the patient-physician interaction. In an article on ethical pitfalls of the EMR, Bernat [4] identified specific issues of copying data with paste functions, perpetuating errors, including inaccurate data in prepopulated templates, and impairment of direct patient-physician communications. Instances of complete notes including physical examination being generated without an actual direct patient encounter are commonplace in the outpatient setting, emergency room, and inpatient consults. Reducing medical errors is central to the purported costsaving of the EMR. Templating operative reports and discharge summaries creates a clear incentive to underreport accurate and specific details of a procedure, and to limit the ability to identify specific risk factors for complications or poor outcome. The EMR template ensures inclusion of all components required for maximal electronic and medical coding without ensuring accuracy or even collection of the data. 


\section{Clinical Faceoff}

Identification of fraud is a priority for federal regulators [6]. The observation of higher charges for emergency room visits is a reflection of higher coding rather than patient illness or intensity of care [15]. The potential for fraud, and the observation of upcoding and billing for services that were not provided is a major risk to the cost saving potential of an EMR.

Dr. Barber: Templates actually enhance our ability to properly record histories, and accurately record procedures and office visits. Having templates prompt you to note a patient's smoking history, or last menstrual period for adolescent girls undergoing surgery will remind orthopedic surgeons about areas they may not focus on otherwise. These prompted bits of history may be critical to patient care.

Templated operative notes often are a real positive for surgeons. How often has a busy total joint surgeon come across an operative dictation that did not include the specifics about the prosthetic that was used? Templated notes will actually provide specific details about the prosthetic being used, including often the lot number and model number. This makes revision surgery down the road easier. The templates also save considerable time, and make the surgeon more productive $[3,11]$.

The potential for fraud exists with any system, and is made more likely not by the system being used but by the culture of providers, and the professionalism that they display. The EMR also makes enforcement easier should a suspicion of fraud be present [12].

Dr. Ries: The EMR provides access for physicians to review labs, radiographs, and other doctor's notes. How will nonacademic or nonhospital-based physicians connect their outpatient EMRs with other physicians, outpatient labs and radiology practices or imaging centers, and the hospital?

Dr. Barber: A big opportunity here comes through Health Information Exchanges. These exchanges will allow physicians and hospitals to exchange information about specific patients. In many areas of the country, these exchanges are up and running and working effectively, but in the majority of the country these exchanges are a work in progress. Many of them have gone broke because of a lack of a solid business model, and others have failed to deliver on their promises. Effective healthcare exchanges are probably a decade away.

The most promising technology for physicians is the "Blue Button" technology. This was developed in the US Department of Veterans Affairs, and is a way to produce an electronic summary of the medical record that can be sent to other providers. This is a simple way to get useful information to the right physician. Many EMR systems are looking to emulate this technology, but common usage is again years away.

Connecting with labs and radiology providers is already here in most EMR systems. It does require some set up and integration with those providers that your office uses, and may not be cheap. This is the area where the most development work has been done, and is the easiest to set up. Connecting with the hospital is very difficult unless you share EMR systems. Sharing of systems is often only possible for larger hospital systems, or large integrated care systems. The future in the arena is very bright, but integration will take time, energy, and effort.

Dr. Berven: Universal access to healthcare information is an important promise of the EMR. The ability of patients and healthcare providers to access specific information within and between healthcare locations offers great potential to improve the rates of information flow. Access to accurate healthcare information will be useful in evaluating a new patient from a remote area, in treating a trauma patient who is unable to share a medical history, and in integrating care between multiple providers. Current EMR systems and health information exchanges have been singularly ineffective in creating effective communication networks. Multicenter research efforts and clinical care have both been limited by inefficiencies and inabilities of health 


\section{Clinical Faceoff}

information exchange networks. The main obstacle to information exchange is the appropriate concern for the security of patient sensitive information and patient privacy. With increased and unregulated healthcare information exchange, patient privacy and personal freedom are placed at significant risk. The paperwork and administrative work generated by breach notifications alone may eliminate much of the promised efficiency of EMR systems.

\section{References}

1. American Academy of Orthopaedic Surgeons Practice Management Committee. EMR: A Primer for Orthopaedic Surgeons, $2^{\text {nd }}$ edition. Rosemont, Illinois: American Academy of Orthopaedic Surgeons; 2010:1-16.

2. Barrows R, Clayton P. Privacy, confidentiality, and electronic medical records. J Am Med Inform Assoc. 1996;3:139-148.

3. Bell B, Thornton K. From promise to reality achieving the value of an EHR. Healthcare Financial Management. 2011;65:51-56.

4. Bernat JL. Ethical and quality pitfalls in electronic health records. Neurology. 2013;80:1057-1061.

5. Blumenthal D, Tavenner M. The "meaningful use" regulation for electronic health records. $N$ Engl $J$ Med. 2010;363:501-504.
6. Carlson J. Warning bell. Potential for fraud through use of EHRs draws federal scrutiny. Mod Healthc. 2012;42: 8-9.

7. Centers for Medicare \& Medicaid Services. EHR Incentive Programs. Available at: http://www.cms.gov/ Regulations-and-Guidance/Legislation/EHRIncentivePrograms. Accessed: October 24, 2013.

8. Congressional Budget Office. Evidence on the costs and benefits of health information technology. 2008. Available at: http://www.cbo.gov/ftp docs/91xx/doc9168/05-20-healthit. pdf - See more at: http://www. ajmc.com/publications/issue/2012/ 2012-8-vol18-n8/which-componentsof-health-information-technology-willdrive-financial-value/4\#sthash.cuNZc 2T3.dpuf. Accessed Oct. 3, 2013.

9. Dimitropoulos L, Patel V, Scheffler SA, Posnack S: Public attitudes toward health information exchange: perceived benefits and concerns. Am J Manag Care. 2011;17:SP111-116.

10. Fernández-Alemán JL, Señor IC, Lozoya PÁ, Toval A: Security and privacy in electronic health records: A systematic literature review. $J$ Biomed Inform. 2013;46:541-562.

11. Jamoom E, Patel V, King J. Furukawa M. National perceptions of EHR adoption: Barriers, impacts, and federal policies. National conference on health statistics. August 2012.

12. Kumar S, Bauer $K$. The business case for implementing electronic health records in primary care settings in the United States. Journal of Revenue and Pricing Management. 2011;10:119-131.

13. Li J, Hurley J, Decicca P, Buckley G. Physician response to pay-forperformance: evidence from a natural experiment [published online ahead of print July 17, 2013]. Health Econ. DOI: 10.1002/hec.2971.

14. Patrikas EO. Electronic databases and privacy protection: issues for a free society. Top Health Inf Manage. 1993;14:62-68.

15. Pitts SR. Higher-complexity ED billing codes-sicker patients, more intensive practice, or improper payments? N Engl J Med. 2012;367: 2465-2467.

16. Shekelle PG, Morton SC, Keeler EB. Costs and benefits of health information technology. Evid Rep Technol Assess (Full Rep). 2006;132: $1-71$.

17. Sidorov J. It Ain't Necessarily So: The Electronic Health Record And The Unlikely Prospect Of Reducing Health Care Costs. Health Aff (Millwood). 2006;25:1079-1085.

18. Siwicki B. Will paper ever go away? Document imagers bet it won't. Health Data Manag. 1995;3:49-50, 52, 54-55.

19. U. S. Department of Health and Human Services. Doctors and hospitals' use of health IT more than doubles since 2012. Available at: http://www.hhs.gov/news/press/2013 pres/05/20130522a.html. Accessed October 25, 2013. 